Quel(s) aspect(s) professionnel(s) apportent le plus de satisfaction aux professeurs de sciences ?

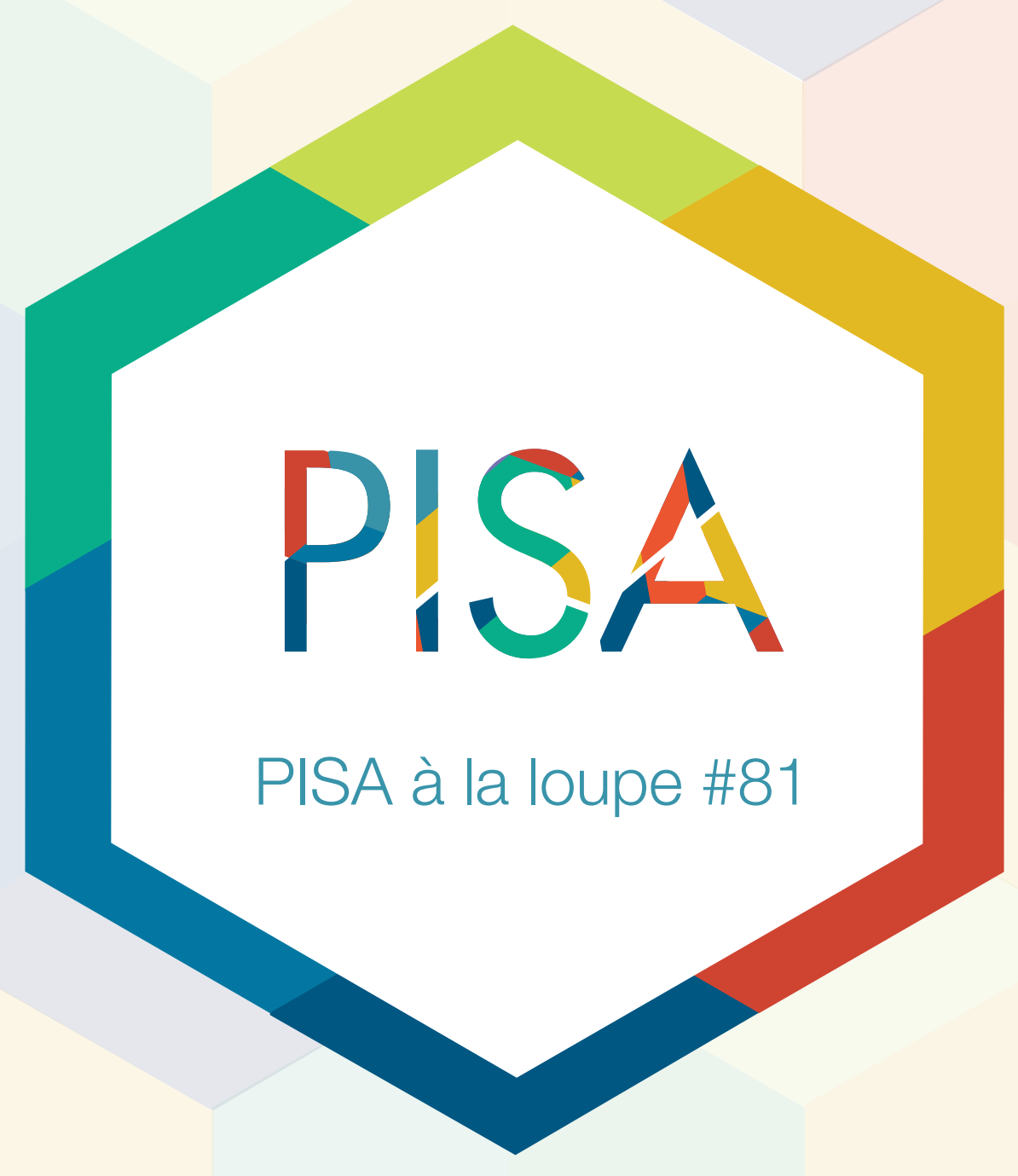




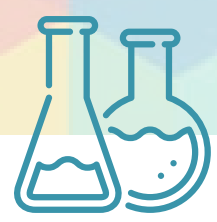

\section{Quel(s) aspect(s) professionnel(s) apportent le plus de satisfaction aux professeurs de sciences?}

- Les professeurs de sciences déclarant qu'à la fin de leurs études secondaires, leur objectif était de devenir enseignant sont plus satisfaits de leur emploi actuel et de leur profession en général que ceux dont ce n'était pas le but.

- Les professeurs de sciences qui collaborent avec leurs collègues et participent à des activités de formation continue se disent plus satisfaits que ceux ne faisant ni l'un ni l'autre.

- La satisfaction des professeurs de sciences à l'égard de leur emploi actuel est fortement associée à l'absence de bruit et d'agitation durant leurs cours, mais pas avec la composition de l'effectif d'élèves de leur établissement en termes de statut au regard de l'immigration et de langue.

Les enseignants jouent un rôle déterminant dans la vie de leurs élèves. Ils leur transmettent le savoir, veillent sur eux, leur servent de modèles et, surtout, créent un environnement propice à l'apprentissage. L'enseignement comporte toutefois de nombreux défis susceptibles d'être source d'insatisfaction ; certains enseignants décident même parfois de quitter définitivement la profession. Dans de nombreux pays du monde entier, les taux élevés de départs de la profession s'avèrent en effet le premier facteur expliquant le manque de professeurs de sciences qualifiés.

De quels aspects de leur travail les professeurs de sciences tirent-ils donc satisfaction, dans une mesure suffisante pour les motiver à poursuivre leur métier d'enseignant en dépit des défis qu'ils peuvent rencontrer?

En 2015, l'enquête PISA a, pour la première fois, administré un questionnaire facultatif aux enseignants des jeunes de 15 ans de 19 pays et économies participants. Ce questionnaire couvre leur environnement de travail, leurs pratiques pédagogiques et leur satisfaction à l'égard de leur emploi actuel et de la profession en général. L'analyse de ces données, combinée aux informations sur leurs élèves et les établissements dans lesquels ils exercent, met au jour des résultats intéressants.

\section{Les professeurs de sciences ayant envisagé leur carrière tôt dans leur parcours comptent en général parmi les plus satisfaits.}

Les professeurs de sciences déclarant qu'à la fin de leurs études secondaires, ils avaient pour objectif de poursuivre une carrière dans l'enseignement se disent plus satisfaits de leur emploi actuel et de la profession d'enseignant en général. Le fait de devenir professeur de sciences est pour eux l'aboutissement d'ambitions nourries de longue date, et non un accident de parcours professionnel. De fait, parmi les différents facteurs considérés dans les analyses, la réalisation de l'objectif de devenir enseignant s'avère l'un des plus fortement associés à la satisfaction des enseignants.

En moyenne, dans tous les pays et économies participants, 58 \% des professeurs de sciences déclarent que leur objectif à la fin du secondaire était de devenir enseignant. Ces professeurs sont bien plus susceptibles de se dire satisfaits de leur métier d'enseignant et de leur emploi actuel. Cette relation est forte dans la plupart des pays et économies, et plus particulièrement au Brésil, aux Émirats arabes unis, dans l'entité de Beijing-Shanghai-Jiangsu-Guangdong (Chine) (ci-après B-S-J-G [Chine]), au Pérou et en République dominicaine.

\section{Des ressources scolaires adéquates et un climat positif contribuent à la satisfaction professionnelle des enseignants.}

La motivation professionnelle ne suffit pas à elle seule lorsque d'autres facteurs font défaut ou entravent le processus d'apprentissage. À titre d'exemple, l'insuffisance des ressources éducatives ou infrastructurelles, ainsi que les problèmes de comportement parmi les élèves d'un établissement, peuvent mettre à mal la satisfaction des enseignants. 


\section{Poursuite d'une carrière dans l'enseignement et satisfaction}

Variation de la satisfaction associée à l'objectif de poursuivre une carrière dans l'enseignement à la fin du <niveau CITE 3>, après contrôle du profil démographique des professeurs de sciences ${ }^{1}$

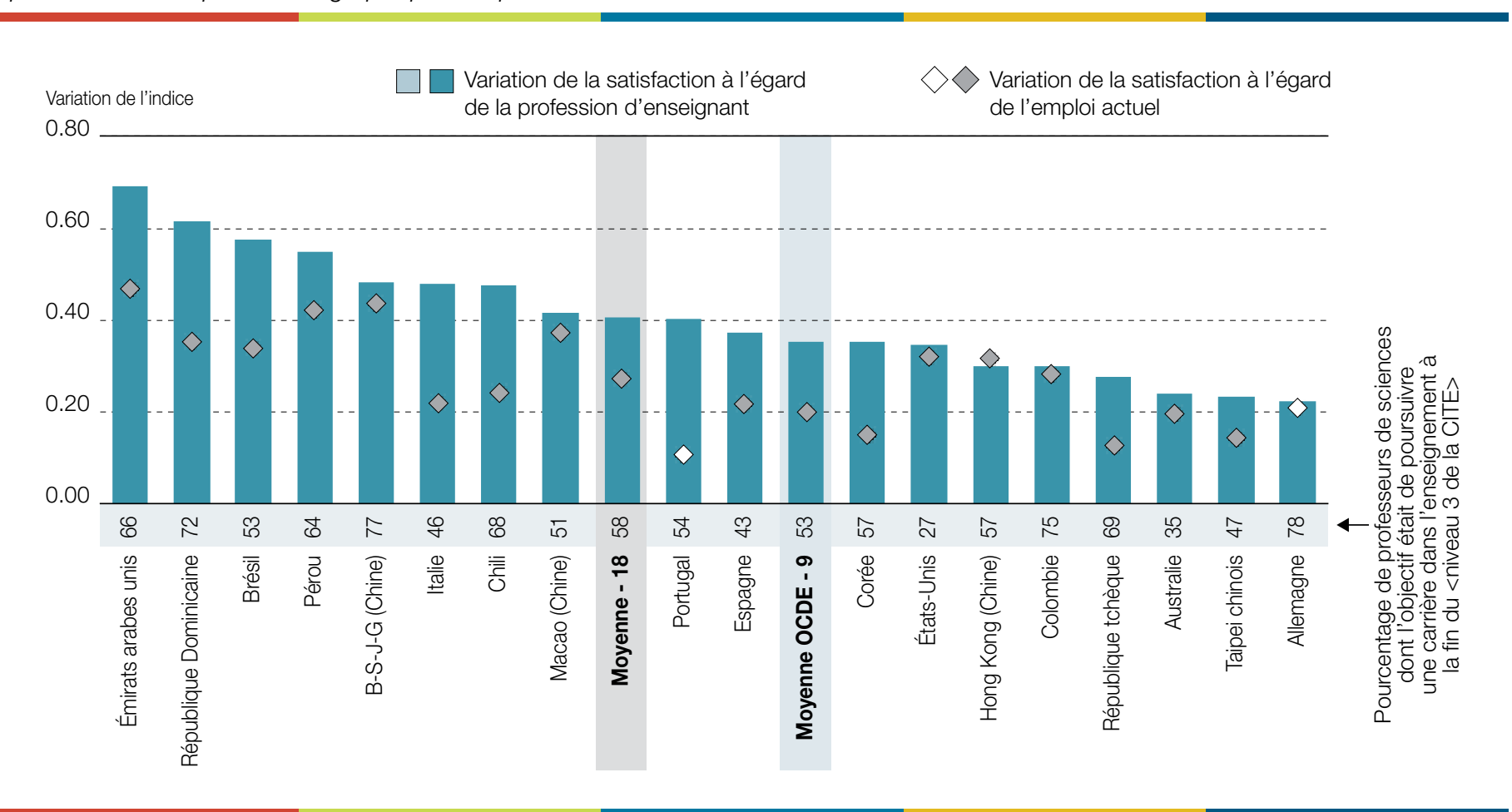

Comment lire ce graphique : Ce graphique montre qu'en moyenne, dans les 18 pays/économies à l'étude, 58 \% des professeurs de sciences des élèves de 15 ans indiquent que leur objectif était de poursuivre une carrière dans l'enseignement à la fin du secondaire (niveau 3 de la CITE). Les enseignants se disant dans ce cas présentent également des niveaux significativement supérieurs de satisfaction à l'égard de la profession d'enseignant et de leur emploi actuel par rapport à leurs collègues dont ce n'était pas l'objectif (comme l'illustrent les différences positives de 0.41 et 0.27 unité sur les indices correspondants), après contrôle des différences de profil démographique des enseignants et de profil socio-économique des établissements.

1. Le profil démographique des professeurs de sciences inclut l'indice PISA de statut économique, social et culturel (SECS) au niveau Établissement, le sexe des professeurs de sciences, le nombre total d'années d'expérience en tant qu'enseignant, le niveau du diplôme le plus élevé obtenu dans l'enseignement institutionnel, le fait d'avoir mené à bien ou non une formation ou des études d'enseignant, et le type de poste dans l'établissement actuel.

Remarque : Les valeurs statistiquement significatives sont indiquées dans une couleur plus foncée.

Les pays et économies sont classés par ordre décroissant de la variation de la satisfaction à l'égard de la profession d'enseignant, après contrôle du profil démographique des professeurs de sciences.

Source : OCDE, Base de données PISA 2015

On observe une association négative entre le manque de ressources humaines et éducatives, tel que communiqué par les enseignants, et la satisfaction de ces derniers. Ainsi, les enseignants qui estiment que le manque de personnel enseignant ou de préparation des enseignants pour leurs cours entrave l'efficacité de leur établissement se disent en général moins satisfaits de leur profession et de leur emploi actuel. La différence de satisfaction entre les enseignants selon qu'ils déclarent que ces types de manques entravent l'efficacité de leur établissement dans une large mesure ou peu, voire pas du tout, est la plus marquée en Allemagne, en Australie, au Brésil, au Chili, aux Émirats arabes unis et à Macao (Chine).

Dans 10 des 18 pays et économies à l'étude, les résultats mettent également au jour l'existence d'une association positive entre la satisfaction des enseignants à l'égard de leur emploi actuel et le climat de discipline en cours de sciences (tel que perçu par les élèves), même après contrôle du profil démographique des professeurs de sciences et du profil socio-économique des établissements. Dans tous les pays et économies participants, l'augmentation d'une unité de l'indice moyen du climat de discipline en cours de sciences au niveau Établissement est associée à une hausse de 0.24 point de l'indice de satisfaction des enseignants à l'égard de leur emploi actuel. Cette association est particulièrement forte en Allemagne, au Brésil, aux États-Unis, au Pérou et en République dominicaine. 


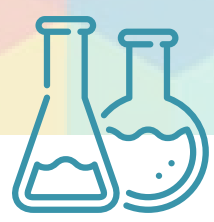

Perception du manque de personnel et satisfaction

Satisfaction des enseignants, par quartile de l'indice de perception, parmi les professeurs de sciences, du manque de personnel

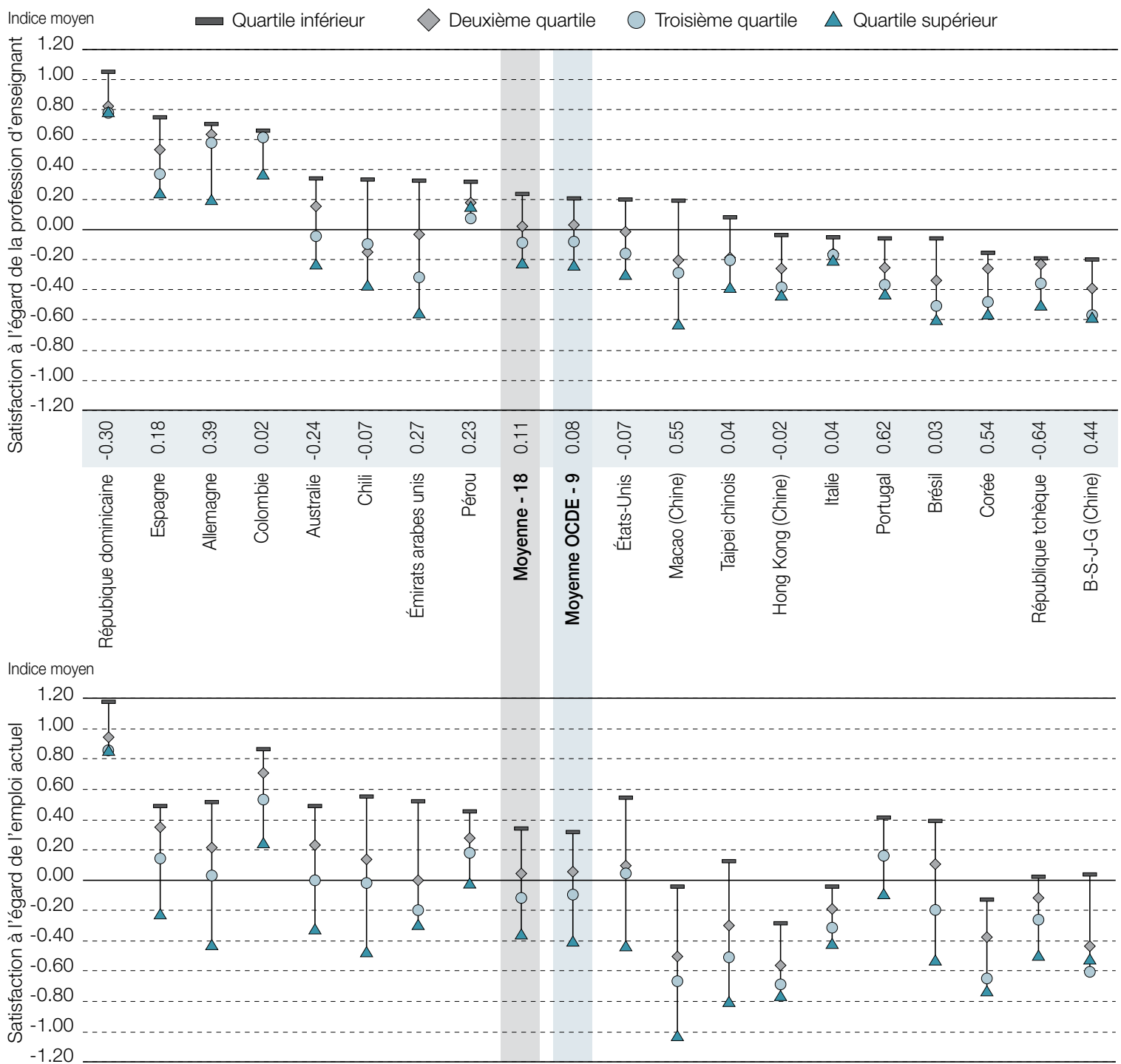

Les pays et économies sont classés par ordre décroissant de la satisfaction à l'égard de la profession d'enseignant chez les enseignants se situant dans le quartile inférieur de l'indice de perception, parmi les professeurs de sciences, du manque de personnel.

Source : OCDE, Base de données PISA 2015.

\section{Une association positive s'observe entre le caractère collaboratif de l'environnement de travail et la satisfaction des enseignants.}

Les enseignants ont plaisir à travailler dans un environnement collégial et bienveillant. D'après les données de l'enquête PISA 2015, les activités de collaboration entre enseignants sont plus courantes en Australie, en Colombie, en Corée, aux Émirats arabes unis, à Macao (Chine), dans l'entité de B-S-J-G (Chine), au Pérou, au Portugal et en République dominicaine, et moins répandues en Allemagne, au Brésil, au Chili, aux États-Unis, en Italie et en République tchèque. Les enseignants faisant part d'activités fréquentes de collaboration entre leurs collègues tendent à être plus satisfaits de leur emploi actuel et de la profession en général. Cette association s'observe dans tous les pays et économies, malgré la variation de l'ampleur des différences entre ces deux groupes d'enseignants. 
Climat de discipline et satisfaction des professeurs de sciences à l'égard de leur emploi actuel Variation de la satisfaction à l'égard de l'emploi actuel associée à l'augmentation d'une unité de l'indice du climat de discipline en cours de sciences au niveau Établissement (tel que rapporté par les élèves)

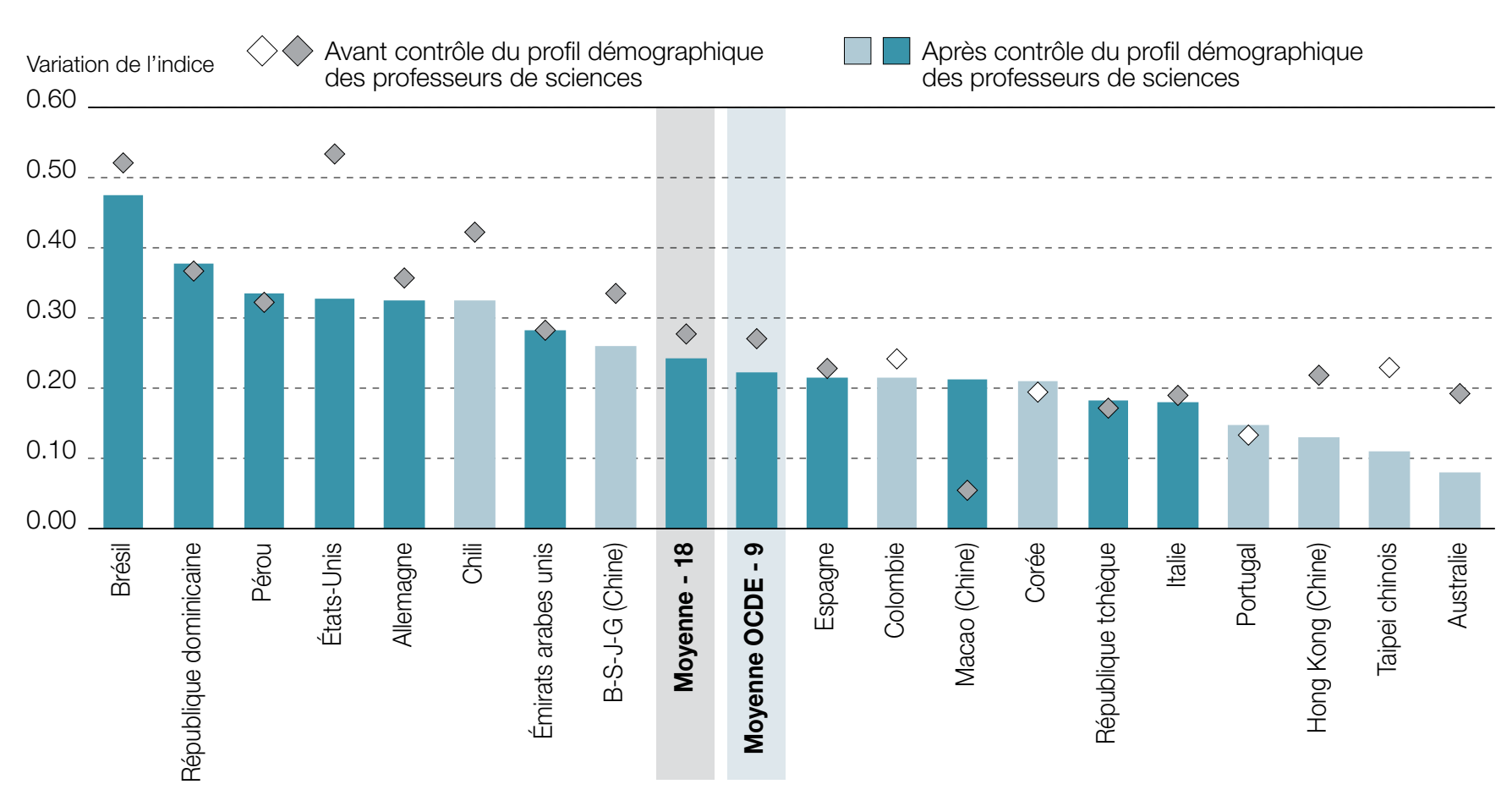

Comment lire ce graphique : Voir les notes sous le graphique 1 "Poursuite d'une carrière dans l'enseignement et satisfaction ».

Remarques : Le profil démographique des professeurs de sciences inclut l'indice PISA de statut économique, social et culturel (SECS) au niveau Établissement, le sexe des professeurs de sciences, le nombre total d'années d'expérience en tant qu'enseignant, le niveau du diplôme le plus élevé obtenu dans l'enseignement institutionnel, le fait d'avoir mené à bien ou non une formation ou des études d'enseignant, et le type de poste dans l'établissement actuel.

L'association avec la satisfaction à l'égard de la profession d'enseignant est plus faible.

Les variables de niveau Élève sont agrégées au niveau Établissement.

Les valeurs statistiquement significatives sont indiquées dans une couleur plus foncée.

Les pays et économies sont classés par ordre décroissant de la variation de la satisfaction à l'égard de l'emploi actuel, après contrôle du profil démographique des professeurs de sciences.

Source : OCDE, Base de données PISA 2015

\section{II existe un lien entre la satisfaction des enseignants et leur participation à des activités de formation continue, mais pas avec le milieu d'origine des élèves de leur établissement.}

La plupart des systèmes d'éducation offrent des possibilités de formation continue à leurs enseignants dans le but d'améliorer leurs compétences, autant d'opportunités également susceptibles de contribuer à renforcer leur confiance en eux et, à terme, leur satisfaction en tant qu'enseignants. D'après l'enquête PISA 2015, les enseignants de la plupart des pays participent à des activités de formation continue. En moyenne, dans tous les pays et économies participants, $52 \%$ des enseignants ont pris part à plus de trois types différents d'activités de formation continue au cours des 12 derniers mois. Leur proportion est particulièrement élevée au Brésil (65\%), aux Émirats arabes unis (65\%), dans l'entité de B-S-J-G (Chine) (82 \%), au Pérou (65\%) et en République dominicaine (76\%).

Les professeurs de sciences ayant participé à plus de trois types d'activités de formation continue au cours des 12 derniers mois sont en général plus satisfaits de la profession d'enseignant et de leur emploi actuel. L'ampleur de l'impact sur ces deux indicateurs de satisfaction est toutefois modérée. 


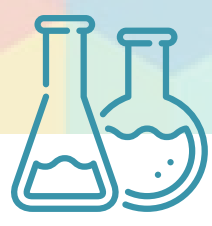

Collaboration entre les professeurs de sciences et satisfaction

Satisfaction des enseignants, par quartile de l'indice de collaboration entre les professeurs de sciences

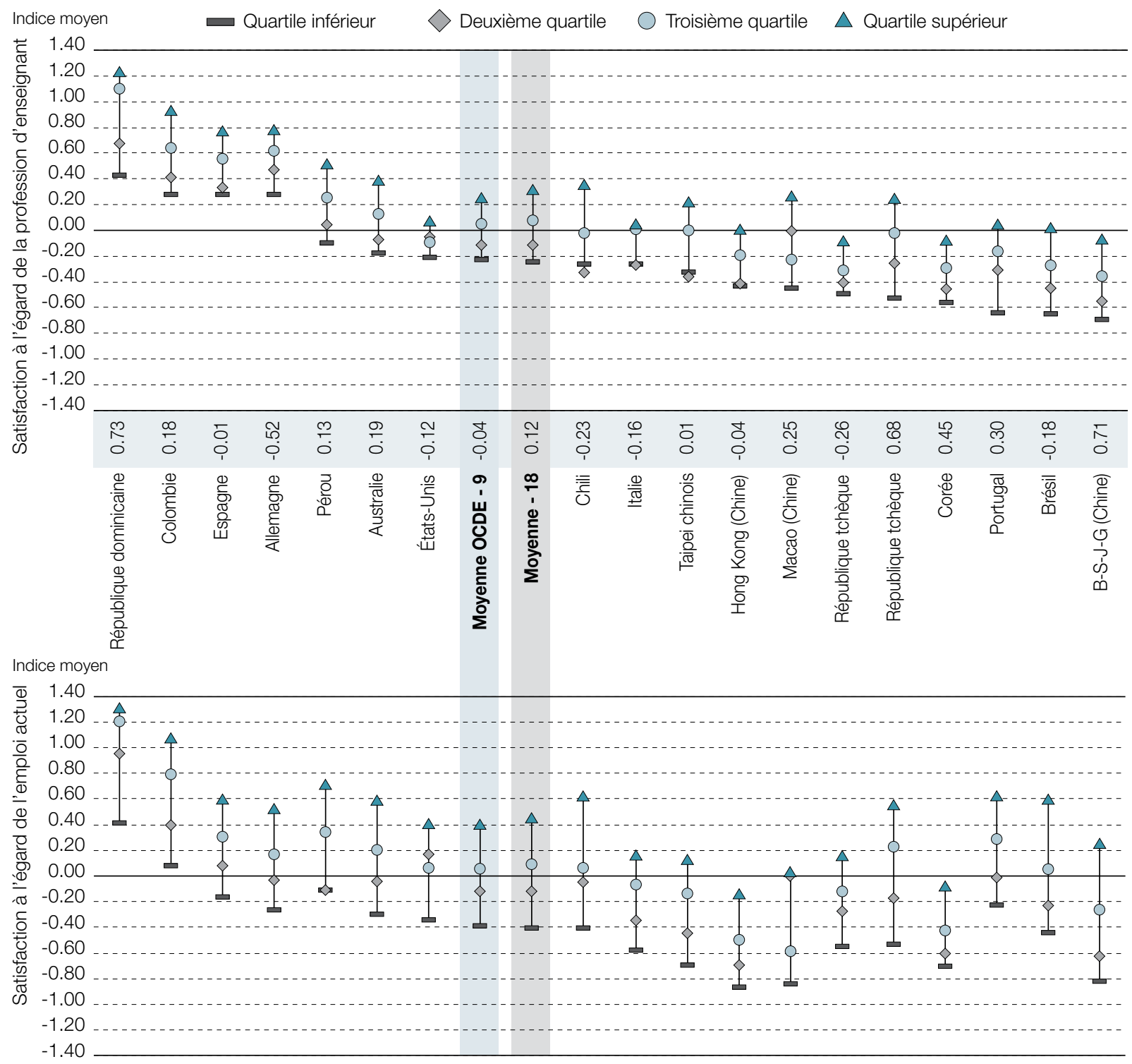

Les pays et économies sont classés par ordre décroissant de la satisfaction à l'égard de la profession d'enseignant parmi les enseignants se situant dans le quartile inférieur de l'indice de collaboration entre les professeurs de sciences.

Source : OCDE, Base de données PISA 2015

Certains facteurs habituellement associés à des environnements d'apprentissage difficiles, tels que la présence de forts pourcentages d'élèves immigrés ou d'élèves ne parlant pas la langue de leur pays d'accueil, ne présentent aucun lien avec le manque de satisfaction des enseignants à l'égard de leur emploi actuel ou de leur profession. Ce constat revêt un intérêt tout particulier, car il montre que les enseignants ne voient pas nécessairement d'inconvénient à enseigner dans des établissements où les élèves sont plus en difficulté tant que l'environnement est propice à l'apprentissage, le climat de l'établissement positif et les ressources adéquates disponibles. II s'agit là d'un constat encourageant, puisqu'il montre que la satisfaction des enseignants dépend de variables que les politiques peuvent faire évoluer. 


\section{Satisfaction des professeurs de sciences à l'égard de la profession d'enseignant,}

selon la participation à des activités de formation continue

Différence de satisfaction des professeurs de sciences à l'égard de la profession d'enseignant, selon qu'ils ont participé à plus/à moins de trois activités de formation continue au cours des 12 derniers mois

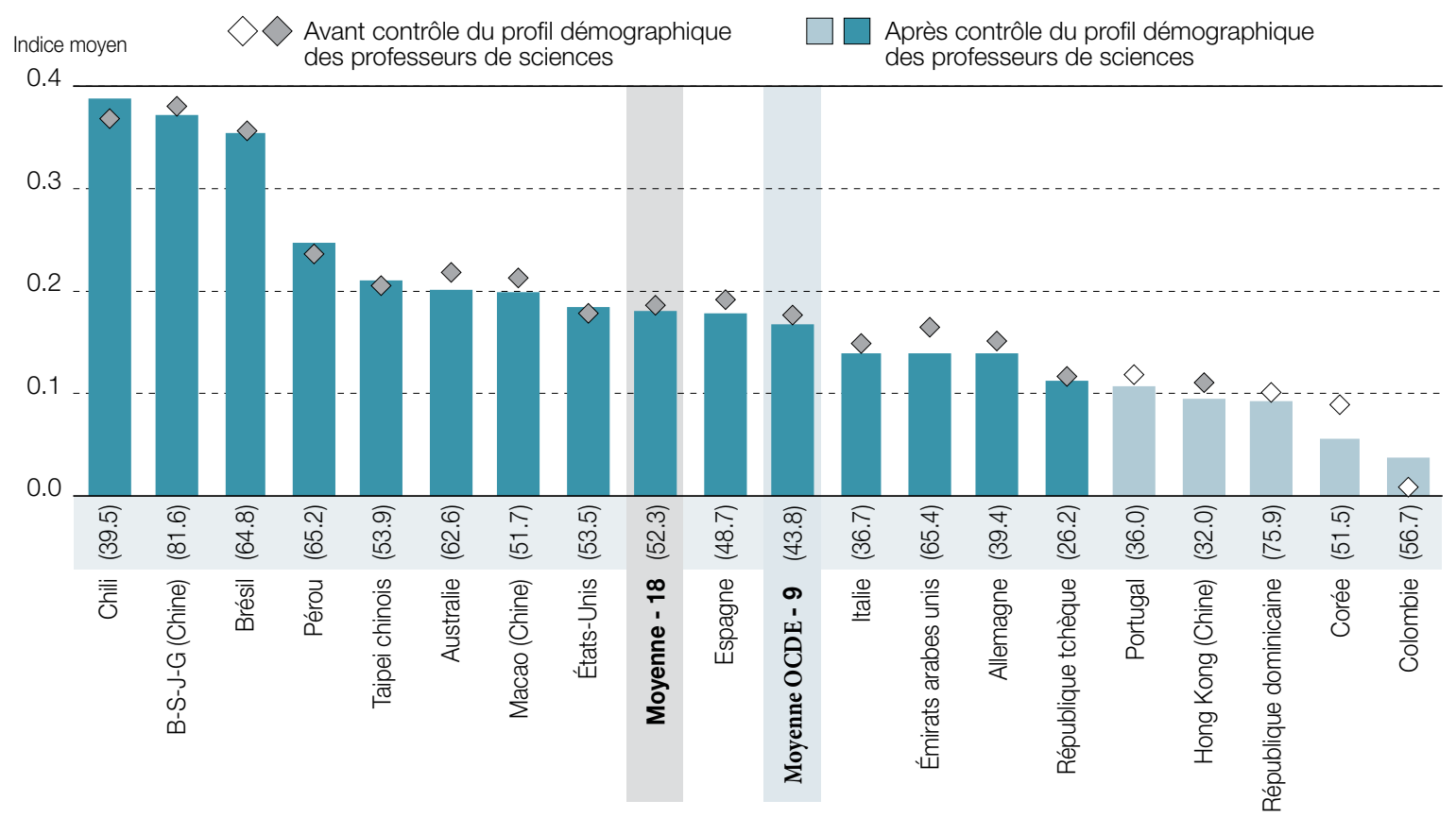

Comment lire ce graphique : Voir les notes sous le graphique 1 «Poursuite d'une carrière dans l'enseignement et satisfaction ".

Remarques : Le profil démographique des professeurs de sciences inclut le sexe, le nombre total d'années d'expérience en tant qu'enseignant, le niveau du diplôme le plus élevé obtenu dans l'enseignement institutionnel, le fait d'avoir mené à bien ou non une formation ou des études d'enseignant, et le type de poste dans l'établissement actuel.

Le pourcentage de professeurs de sciences ayant participé à plus de trois activités de formation continue au cours des 12 derniers mois est indiqué en regard du nom des pays/économies.

Les valeurs statistiquement significatives sont indiquées dans une couleur plus foncée.

Les pays et économies sont classés par ordre décroissant de la différence de satisfaction des professeurs de sciences à l'égard de la profession d'enseignant selon qu'ils ont participé à plus/à moins de trois activités de formation continue au cours des 12 derniers mois, après contrôle du profil démographique des professeurs de sciences.

Source : OCDE, Base de données PISA 2015

\section{Pour conclure}

Une association positive s'observe entre la satisfaction des enseignants et différents facteurs connus pour améliorer la performance des élèves, tels que l'accès à un environnement scolaire collégial et positif. En d'autres termes, la satisfaction des enseignants à l'égard de leur emploi actuel et de leur profession constitue à la fois une composante et une conséquence de l'environnement scolaire. L'amélioration de l'expérience d'apprentissage de l'ensemble des élèves devrait par conséquent aussi renforcer la satisfaction professionnelle de leurs enseignants. 


\section{Pour tout complément d'information}

Contacter : Tarek Mostafa (tarek.mostafa@oecd.org)

Consulter : Mostafa, T. et J. Pál, Science teachers' satisfaction: Evidence from the PISA 2015 teacher survey,

Documents de travail de l'OCDE sur l'éducation, n 168, http://dx.doi.org/10.1787/19939019.

Le mois prochain : Performance et bien-être des élèves issus de l'immigration : comment se situent les différents pays ?

Cet ouvrage est publié sous la responsabilité du Secrétaire général de l'OCDE. Les opinions et les interprétations exprimées ne reflètent pas nécessairement les avis des pays membres de l'OCDE.

Ce document, ainsi que les données et cartes qu'il peut comprendre, sont sans préjudice du statut de tout territoire, de la souveraineté s'exerçant sur ce dernier, du tracé des frontières et limites internationales, et du nom de tout territoire, ville ou région.

Ce texte est disponible sous licence Attribution - Pas d'Utilisation Commerciale - Partage dans les Mêmes Conditions 3.0 Organisations Internationales

(CC BY-NC-SA 3.0 IGO). Pour toute information spécifique quant à l'étendue et aux termes de la licence ainsi que d'une possible utilisation commercial de ce texte et pour toute usage de données PISA, prière de consulter les Conditions d'utilisation à http://www.oecd.org/fr/conditionsdutilisation. 